\title{
Reconnecting Broken Ridges in Fingerprint Images
}

\author{
Nadia Brancati, Maria Frucci, and Gabriella Sanniti di Baja \\ Institute of Cybernetics “E. Caianiello", CNR, Pozzuoli (Naples), Italy \\ n.brancati@cib.na.cnr.it, m.frucci@cib.na.cnr.it, \\ g.sannitidibaja@cib.na.cnr.it
}

\begin{abstract}
In this paper, we present a new method for reconnecting broken ridges in fingerprint images. The method is based on the use of a discrete directional mask and on the standard deviation of the gray-levels to determine ridge direction. The obtained direction map is smoothed by counting the occurrences of the directions in a sufficiently large window. The fingerprint image is, then, binarized and thinned. Linking paths to connect broken ridges are generated by using a morphological transformation to guide the process.
\end{abstract}

\section{Introduction}

Fingerprints are widely used in biometric techniques for automatic personal identification, though a number of other techniques exist, which involve other biometric features such as face, iris, ear and voice. In fact, fingerprints of any individual are unique (even in the case of identical twins), remain the same over lifetime, and are easy to collect.

Fingerprints were initially introduced for criminal investigation, and their verification used to be performed manually by experts. Nowadays fingerprints are still used to identify suspects and victims of crimes, but are also involved in an increasing number of applications, such as physical access control, employee identification, and information systems security. Moreover, the tedious manual matching work has been replaced by automatic fingerprint identification systems, which can work with databases including even several millions records of fingerprint images. Most of the fingerprint matching algorithms follow the forensic procedure of matching particular features in a fingerprint image, called minutiae. The minutiae are local discontinuities of ridgelines in a fingerprint image. Though minutiae could be classified in several classes, it is standard practice to use a classification in two minutiae only, namely termination and bifurcation.

Fingerprints can be captured by different devices, each of which may produce a corrupted image. For automatic fingerprint identification, the quality of fingerprint images is of great importance, since low quality images severely affect the detection of the minutiae and, accordingly, a correct identification. For this reason, fingerprint images generally undergo a number of different processes, aimed at enhancement, computation of the ridge direction, thinning, and feature extraction.

Enhancement largely contributes to the robustness of a system for fingerprint verification/recognition and is a topic that has received much attention. In this paper, we focus on the problem of reconnecting broken ridges. We suggest a method based on 
the use of a discrete $9 * 9$ directional mask and on the standard deviation of the graylevels to build an initial direction map. The final direction map is, then, obtained by counting the occurrences of the directions within a $45 * 45$ window. The skeleton of the binarized fingerprint image is used, together with the watershed transform, computed by taking into account the end points and the isolated pixels of the skeleton, to generate linking paths and reconnect broken ridges.

\section{Related Works}

Enhancement of damaged fingerprint images has received much attention in the literature. Different techniques based, for example, on the use of Gabor filter, directional median filter, thresholding, wavelets, were suggested to improve the quality of the gray level fingerprint image [1-4]. Most of the existing methods can be classified in two categories, depending on whether frequency domain or spatial domain is considered.

In the frequency domain, enhancement methods are mainly based on the Fourier transform [5-7]. For example, the method suggested in [5] uses a bandpass filter to build 16 images filtered in 16 different directions. For each image, the inverse Fourier transform is computed and the 16 obtained images are suitably combined to get an enhanced image. In [7], enhancement is obtained without resorting to information derived from the directions along the ridges. The fingerprint image is divided into non-overlapping blocks of size $32 * 32$ and the Fourier transform of each block is computed. The underlying idea is that the direction along the ridge is constant within each block. A drawback common to enhancement methods based on the Fourier transform is that short ridges are often filtered out. Thus, ridges that are broken in many small parts are not always reconstructed.

In the spatial domain, ridge directions are extracted from the gradient of the fingerprint image (see e.g., [8-10]), or by means of directional masks (see e.g., [11-14]). In [9], a bandpass filter is used to filter the image in 2 directions so as to reduce noise before computing the gradient. In [14], the fingerprint image is divided into non overlapping blocks, the direction of the central pixel is computed by using a directional mask and this direction is assigned to all pixels of the block, so generating a smoothed direction map. In [15], 16 directions are considered and the standard deviation of gray-levels of pixels aligned along them is computed. The direction to be assigned to each pixel $p$ is found by selecting the pair of perpendicular directions for which the highest discrepancy is found: if the two standard deviations differ, the direction of $p$ is the one with the smaller standard deviation, otherwise a special value is assigned to $p$. The basic idea is that along a ridge the standard deviation should be remarkably smaller than that in the perpendicular direction. Since pixels of the fingerprint image in correspondence with broken ridges are generally assigned the special value, windows of different sizes are used to compute the standard deviations and update the direction map. Gradient-based method may fail to detect correct directions in the ridge breakings areas. Methods based on directional masks to compute ridge direction and on the subdivision of the fingerprint image into non-overlapping blocks to get a smoothed direction map have the same drawback, since the direction map updating is done in a too global way. Methods like [15], where the direction map updating is done with local criteria, are more effective. The main problem is the selection of the size of the windows used for the updating. 


\section{The Suggested Method}

Our method for reconnecting broken ridges is in the class of methods working in the spatial domain and on the use of directional masks. Our work has been inspired by the method suggested in [15] as concerns the construction of the direction map. The method has been tested on the fingerprint images used for the 'Fingerprint Verification Competition'(FVC) of years 2000, 2002 and 2004.

\subsection{Building the Direction Map}

Working in the discrete plane, the number of possible directions depends on the size of the selected directional mask. For a size $n^{*} n, 2 * n-2$ directions are possible. In turn, the size of the directional mask should be selected by taking into account the thickness of the fingerprint ridges and of the valleys separating them. If a too small mask is centered on the innermost pixels in a ridge (valley), only very small gray-level changes would be detected along all directions, so that the innermost pixels could not be assigned any direction. In turn, if a too large mask is used, wherever the mask hits a portion of the image including both ridges and valleys, quite high gray-level variations would be detected, causing non-correct direction assignment.

We use, a directional mask of size $9 * 9$, which is a reasonably good compromise for our dataset and, hence, 16 different directions result to be possible. The discrete directional mask is given in Fig. 1. A darker tone is used to identify the lines passing through the pixels along the direction in one of the two ways (positive) and a lighter tone to identify the lines in the opposite (negative) way.

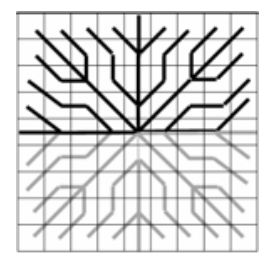

Fig. 1. The $9 * 9$ discrete directional map

Our criterion to build the direction map is improved with respect to the one in [15]. For each pixel $p$ in the fingerprint image, the standard deviation of the gray-levels of the pixels aligned along each of the above directions is computed in a $9 * 9$ window. Let $\min$ be the minimum value of the standard deviation. The absolute value of the difference in standard deviation for each pair of perpendicular directions and the maximal value, $\max$, of such a difference are also computed.

In principle, for a pixel $p$ located along a ridge (valley) the standard deviation along the ridge (valley) direction should be the only one with value min. If this is the case, the unique direction with standard deviation $\min$ is by all means the direction to be assigned to $p$. However, when the ridge (valley) includes pixels with scarcely different gray-levels, the standard deviation can be equal to min in more than one direction and some ambiguity in the direction selection exists. We distinguish two cases. If the standard deviation is equal to $\min$ in all the principal (horizontal, vertical and 

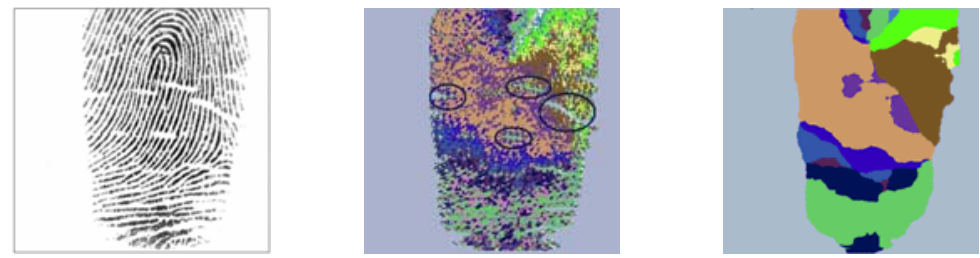

Fig. 2. A fingerprint, left, the initial direction map, middle, and the final direction map, right

diagonal) directions, we consider the direction for $p$ as undetermined and assign to $p$ a special value $v$ in the direction map. Otherwise, we select the pair of perpendicular directions for which the absolute value of the difference in standard deviation is equal to max. If the difference max is found for only one pair of perpendicular directions, the direction characterized by the smaller value in that unique pair is assigned to $p$. Otherwise, a decision on the direction for $p$ cannot be taken, and $p$ is assigned the special value $v$ in the direction map.

As an example, in Fig. 2 the fingerprint used as running example in this paper is shown to the left, and the computed initial direction map is given in the middle. Not all pixels of the image have been assigned one of the possible directions, some of them are assigned the special value $v$. In particular, the special value $v$ is assigned not only to pixels that, actually, are not part of the fingerprint, but also to all pixels in the zones, circled in Fig. 2 middle, corresponding to the ridge breaking areas. Thus, no direction information is available in the ridge breaking areas; moreover, the initial direction map shows a too complex distribution of directions to be easily used, and a suitable updating of the direction map is necessary.

The updating of the direction map is done, as suggested in [15], by using a $45 * 45$ window. For each pixel $p$, we count in the window the occurrences of each direction and assign to $p$ the direction with the maximal occurrence. The final direction map can be seen in Fig. 2, right. Almost all pixels of the fingerprint have been assigned a direction and the obtained final direction map is noticeably smoother than the initial map. The final direction map may include less directions with respect to those in the initial map.

\subsection{Binarization and Thinning}

Rather than directly connecting broken ridges in the fingerprint image, we resort to skeletonization to thin down the ridges to unit width and suggest a way to link to each other thin lines found in correspondence of broken ridges.

A preliminary thresholding of the fingerprint image is performed to distinguish the ridges (foreground) from the background. To this purpose, a pixel $p$ is taken as a foreground pixel if its gray-level is smaller than or equal to the average gray-level in a $9 * 9$ window centered on $p$. Otherwise, $p$ is taken as a background pixel. Skeletonization is accomplished by using the algorithm [16], which also includes a postprocessing phase to prune non-significant branches and to reduce the zig-zags created during reduction to unit width. For the running example, the result of skeletonization is shown in Fig. 3, where the binarized fingerprint image is shown to the left and the resulting skeleton in the middle. 

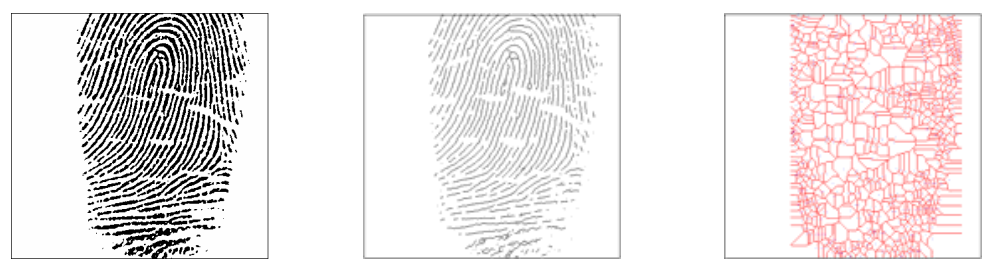

Fig. 3. The binarized fingerprint, left, the skeleton, middle, and the watershed transform, right

\subsection{Linking Paths Guided by the Watershed Transform}

Skeleton parts found in correspondence of broken ridges consist of a number of components. To link these components, we need to build linking paths connecting their extremes (end points of skeleton branches or isolated pixels of the skeleton). Naturally, not all skeleton components have to be linked to each other, which implies a selection of a proper subset of extremes, called significant extremes. To this purpose, we use the watershed transform [17-19].

The computation of the watershed transform of an image implies the detection of a set of pixels in the image, the seeds, from which to perform region growing. Region growing associates to each seed $s$ those image pixels that are closer to $s$ than to any other seed. In our case, we compute the watershed transform of the binary image, where the seeds, i.e., the extremes of all skeleton components, have value 1, and all remaining pixels have value 0 . The growing process provides a partition of the image, where each region surrounded by watershed lines represents the area of influence of a seed. See Fig. 3 right. In this way, in correspondence of the ridge breaking areas, the watershed lines will be mostly placed midway between the extremes of skeleton components that we would like to connect, and the watershed lines can be seen as the barriers where the linking paths starting from the extremes terminate. Depending on the oriented directions of pairs of paths terminating on the same watershed line, and on the distance between the termination points of the paths, we can decide whether the extremes from which the paths originate are significant extremes.

To add to the skeleton the path associated to each pixel $p$ that is one of the extremes, the direction associated to $p$ in the final direction map is taken into account. Obviously, if $p$ is an isolated pixel, two paths in are built in the two opposite ways of the direction, while if $p$ is an end point, a unique path is built, which is the prolongation of the skeleton branch in the direction associated to $p$. The $9 * 9$ discrete directional map shown in Fig. 1 is centered on the pixel $p$ and all pixels along the direction associated to $p$ in the appropriate way(s) are assigned to the path. If any of the pixels ascribed to the path belongs to a watershed line, path building from $p$ is interrupted at that pixel, since the termination point of the path has been reached. Otherwise, the $9 * 9$ window is centered on the last pixel ascribed to the path, and the path building process is repeated starting from it, still in the direction of $p$.

Initially, a list of the end points is constructed and only paths built from the end points are considered. To decide whether $p$ is a significant end point and, hence, the path built from it, path_p, has to be actually used for broken ridge connection, we analyze $p a t h \_p$ and any other path, path $\_q$, originated from other extremes and terminating on the same watershed line. Two main cases are possible for the termination 
point of the path associated to $p$, which can be a simple termination point, or a complex termination point. A termination point is simple, white circles in Fig. 4, if it is adjacent to only two regions of the watershed partition. A termination point is complex, black circles in Fig. 4, when the termination point is adjacent to more than two regions. A different criterion is used to decide if path_p has to be taken or has to be removed, depending on whether $q$ is an end point or an isolated pixel.
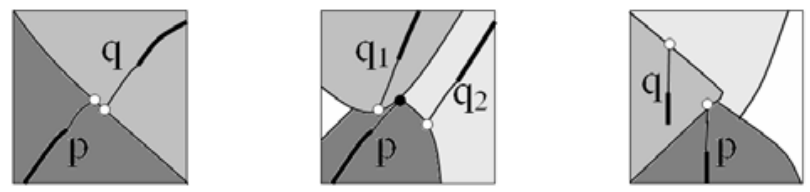

Fig. 4. Simple termination points (white circles) and complex termination points (black circles)

Let us suppose that the termination point for path_p is simple and that the path has been built in positive way. The termination point of path_p separates only two areas of influence, which are associated to $p$ and to $q$, (see Fig. 4 left). The procedure is different depending on whether $q$ is an end point or an isolated pixel. In the first case, if path_q has been built in negative way, and the distance between the termination points of $p a t h \_p$ and $p a t h \_q$ is smaller than an a priori fixed threshold $\theta$, both path_p and path_q are interpreted as necessary to connect a broken ridge; if the distance overcomes the threshold, $p$ is regarded as not placed in a ridge breaking area; finally, if also path $\_q$ has been built in positive way, decision on the significance of path $\_p$ can not yet be taken. An example in which $p a t h \_q$ and $p a t h \_p$ are built in the same way is shown Fig. 4 right, where the two paths path_p and path_q were built in correspondence with skeleton components belonging to two mostly parallel ridges that should not be reconnected. In the case that $q$ is an isolated pixel, the criterion to establish if $p$ and $q$ should be linked to each other is the following: only if $q$ is located along the same discrete direction associated to $p$, the two pixels $p$ and $q$ have to be linked to each other. If this is the case, linking occurs by taking path $p$ and by prolonging it to reach $q$, along the direction assigned to $p$. If $q$ is not along the discrete direction associated to $p$, the decision on the significance of path $p$ can not yet be taken.

Let us now suppose that the termination point for $p a t h \_p$ is complex. The termination point of path $p$ separates more than two areas of influence, which are associated to $p$ and to $q_{1}, q_{2}$, etc. (see Fig. 4 middle). A decision must be taken to select, among the paths, path_q $q_{1}$, path_q $q_{2}$ etc., terminating on the crossing watershed lines, which one should be possibly accepted together with path_p. Paths generated by end points $q_{i}$ are considered first. Only paths, built in opposite way with respect to path_p and such that the distance between their termination points and the termination point of $p a h \_p$ is under the threshold $\theta$, are taken into account. Among them, the path with the minimal distance is taken, together with $p a t h \_p$, for reconnecting a broken ridge. Of course, if no path_q $q_{i}$ in opposite way exists or the threshold is overcome, the $q_{i}$ that are isolated pixels, if any, are considered and the same procedure followed in case of isolated pixels and simple termination point is followed. 

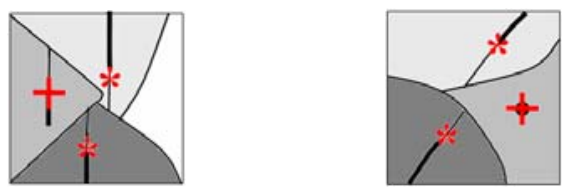

Fig. 5. The regions associated to extremes that should be connected, stars, are not adjacent

Whenever linking paths are accepted as necessary for reconnecting ridges, an updating of the list of the end points is accomplished. In fact, when an end point is linked to an isolated pixel, a new end point, coinciding with the isolated pixel, is generated in the skeleton, from which a new path can be built. The process of path building and identification of significant paths is iterated as far as new end points are added to the list.

The above process works correctly if the partition regions associated to two significant extremes are adjacent. However, this is not always the case. For example, the extremes denoted by stars in Fig. 5 should be linked to each other, but the corresponding partition regions are separated by a partition region associated to the extreme denoted by a cross, with whom (correctly!) linking was not accomplished during the above process.

A second step of path building, allowing a path to cross the barriers and continue until the watershed line of the next partition region is met, is necessary. Also the second step is performed starting only from the end points, and the end points that are taken into account are those that during the previous phase were not classified as significant extremes, i.e., a decision on their associated paths was not taken. The second step is done by using the same process accomplished in the first step, the only difference being that the areas of influence of the pixels that we are going to link are not adjacent. Isolated pixels of the skeleton possibly remaining at the end of the reconnection process are disregarded and are considered as noise.

\subsection{Results and Conclusion}

The performance of our method, when applied to the datasets used for FVC, has been evaluated by an expert of the Police Headquarters of Naples, Italy, who found the results very good for a more reliable identification of the minutiae. For space limitation, in Fig. 6 only four images with reconstructed ridges are shown, where the image to left is for the fingerprint used as running example.
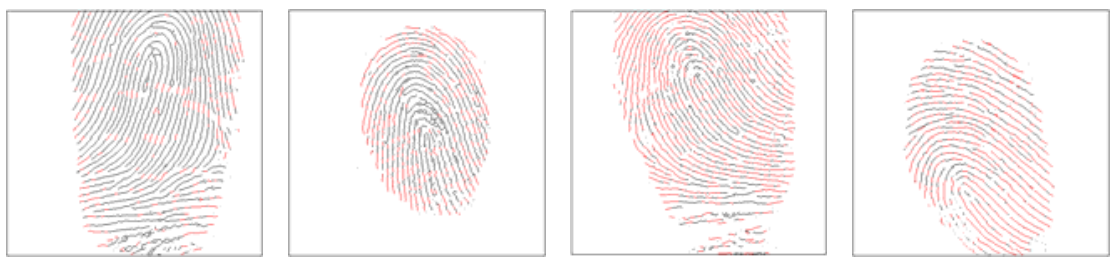

Fig. 6. Reconstructed ridges (red color denotes reconstructed pixels) 

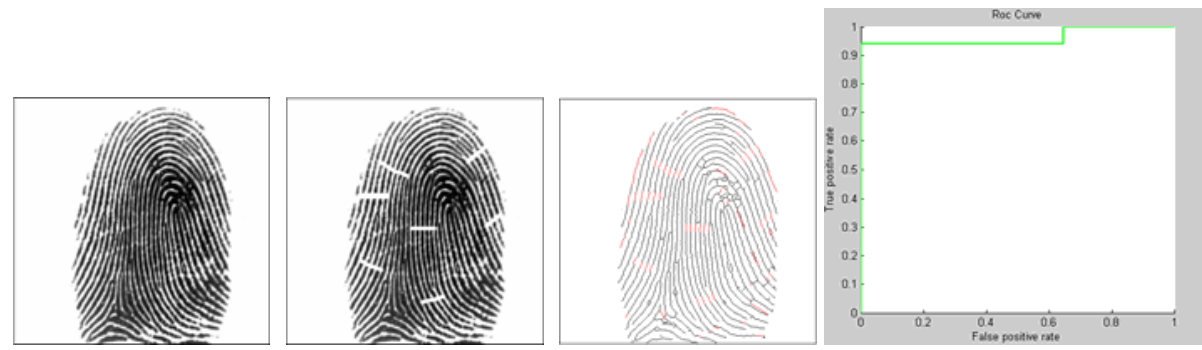

Fig. 7. From left to right: a fingerprint, its artificially corrupted version, the fingerprint reconstructed by using a $45 * 45$ window for updating the direction map, and the ROC curve

To test the method also for images largely corrupted, we have artificially increased the number of ridge breaking areas in the fingerprints of the dataset. The original images have been compared with the results obtained by processing the artificially corrupted images to verify the ability of our method in reconnecting broken ridges. Windows of different size, ranging from $9 * 9$ to $73 * 73$, have been used for the computation of the final direction map in order to build a ROC curve and so evaluate the goodness of the method. About 50 artificially corrupted images have been used and in all cases, the area under the ROC curve is larger than 0.90, indicating the effectiveness of the proposed method. Again for space limitation, only one example is given in Fig. 7.

It might appear that, besides the analogy as concerns the construction of the direction map, yet another analogy exists between our method and the one in [15] since both methods use the watershed transform. However, we stress that the watershed transform is used for different aims in the two methods. For [15], the watershed transform of the fingerprint image is used to associate partition regions with valleys and ridges. Then, two direction maps are computed, for the fingerprint image and for the watershed transform, and the directions in these two maps that are perpendicular to each other are used to identify the ridge breaking areas. In our case, instead, the watershed transform is only used to guide the path building process.

Our method has been implemented in $\mathrm{C}$ on a standard PC, by using OpenCV libraries. Future work will be done to improve the construction of the direction map, especially as concerns pixels placed at peripheral parts of the fingerprint.

\section{Acknowledgements}

We are gratefully indebted to Dr. Luigi Bisogno, Head Detective Inspector, Department of Judicial Dactyloscopy of the Scientific Police, Police Headquarters of Naples, for his precious help in evaluating the results of our method.

\section{References}

[1] Wu, C., Shi, Z., Govindaraju, V.: Fingerprint image enhancement method using directional median filter. In: Proc. SPIE 2004, vol. 5404, pp. 66-75 (2004)

[2] Hong, L., Wan, Y., Jain, A.: Fingerprint image enhancement: algorithm and performance evaluation. IEEE Trans. PAMI 20(8), 777-789 (1998) 
[3] Areekul, V., Watchareeruetai, U., Suppasriwasuseth, K., Tantaratana, S.: Separable Gabor filter realization for fast fingerprint enhancement. In: Proc. ICIP 2005, vol. 3, pp. 253-256 (2005)

[4] Yang, J., Liu, L., Jiang, T., Fan, Y.: A modified Gabor filter design method for fingerprint image enhancement. Pattern Recognition Letters 24(12), 1805-1817 (2003)

[5] Sherlock, B.G., Monro, D.M., Millard, K.: Fingerprint Enhancement by Directional Fourier Filtering. IEEE Proc. - Visual Image Signal Processing 241(2), 87-94 (1994)

[6] Ikonomopoulos, A., Unser, M.: A Directional Filtering Approach to Texture Discrimination. In: Proc. Seventh International Conference on Pattern Recognition, pp. 87-89 (1984)

[7] Willis, A.J., Myers, L.: A Cost-Effective Fingerprint Recognition System for Use with Low-Quality Prints and Damaged Fingertips. Pattern Recognition 34, 255-270 (2001)

[8] Jain, A.K., Lin, H., Bolle, R.: On-line Fingerprint Verification. IEEE Transactions on Pattern Analysis and Machine Intelligence 19(4), 302-314 (1997)

[9] Hong, L.: Automatic Personal Identification Using Fingerprints, Ph.D Dissertation (1998)

[10] Rao, A.: A Taxonomy for Texture Description and Identification. Springer, Heidelberg (1990)

[11] Stock, R.M., Swonger, C.W.: Development and Evaluation of a Reader of Fingerprint Minutiae, Technical Report CAL No. XM-2478-X-1, pp. 13-17 (1969)

[12] Candela, G.T., Grother, P.J., Watson, C.I., Wilkinson, R.A., Wilson, C.L.: PCASYS - A Pattern-Level Classification Automation System for Fingerprints, Technical Report (1995)

[13] Eun-Kyung, Y., Sung-Bae, C.: Adaptive Fingerprint Image Enhancement with Fingerprint Image Quality Analysis. Image and Vision Computing 24, 101-110 (2006)

[14] Madhusoodhanan, P., Sumantra Dutta, R.: Robust Fingerprint Classification Using an Eigen Block Directional Approach. In: Indian Conference on Computer Vision, Graphics and Image Processing, ICVIGIP 2004 (2004)

[15] Oliveira, M.A., Leite, N.J.: A Multiscale Directional Operator and Morphological Tools for Reconnecting Broken Ridges in Fingerprint Images. Pattern Recognition 41(1), 367-377 (2008)

[16] Sanniti di Baja, G., Thiel, E.: Skeletonization Algorithm Running on Path- Based Distance Maps. Image and Vision Computing 14, 47-57 (1996)

[17] Roerdink, J.B.T.M., Meijster, A.: The Watershed Transform: Definitions. Algorithms and Parallelization Strategies, Fundamenta Informaticae 41, 187-228 (2001)

[18] Beucher, S., Meyer, F.: The Morphological Approach to Segmentation: the Watershed Transformation. In: Mathematical Morphology in Image Processing, ch. 12, pp. 433-481. Marcel Dekker, New York (1992)

[19] Meyer, F.: Color Image Segmentation. In: Proceedings of the International Conference on Image Processing and its Applications, pp. 303-306 (1992) 